\title{
A 4'-Phosphopantetheinyl Transferase Mediates Non-Ribosomal Peptide Synthetase Activation in Aspergillus fumigatus
}

\author{
Claire Neville, Alan Murphy, Kevin Kavanagh, and Sean Doyle ${ }^{*^{[a]}}$
}

\begin{abstract}
Aspergillus fumigatus is a significant human pathogen. Non-ribosomal peptide (NRP) synthesis is thought to be responsible for a significant proportion of toxin and siderophore production in the organism. Furthermore, it has been shown that 4'-phosphopantetheinylation is required for the activation of key enzymes involved in non-ribosomal peptide synthesis in other species. Here we report the cloning, recombinant expression and functional characterisation of a $4^{\prime}$-phosphopantetheinyl transferase from A. fumigatus and the identification of an atypical NRP synthetase (Afpes 1), spanning $14.3 \mathrm{~kb}$. Phylogenetic analysis has shown that the NRP synthetase exhibits greatest identity to NRP synthetases from Metarhizium anisolpiae (PesA) and Alternaria brassicae (AbrePsy1). Northern hybridisation and RT-PCR analysis
\end{abstract}

have confirmed that both genes are expressed in A. fumigatus. A $120 \mathrm{kDa}$ fragment of the A. fumigatus NRP synthetase, containing a putative thiolation domain, was cloned and expressed in the baculovirus expression system. Detection of a 4'-phosphopantetheinylated peptide (SFSAMK) from this protein, by MALDI-TOF mass spectrometric analysis after coincubation of the 4'-phos phopantetheinyl transferase with the recombinant NRP synthetase fragment and acetyl CoA, confirms that it is competent to play a role in NRP synthetase activation in A. fumigatus. The 4'-phosphopantetheinyl transferase also activates, by 4'-phosphopantetheinylation, recombinant $\alpha$-aminoadipate reductase (Lys2p) from Candida albicans, a key enzyme involved in lysine biosynthesis.

\section{Introduction}

Aspergillus fumigatus is a significant human pathogen, particularly in immunocompromised individuals. ${ }^{[1]}$ Three forms of aspergillosis are recognised clinically: saprophytic, allergic and invasive. Invasive aspergillosis (IA) is the most serious form of disease as it involves the invasion of viable tissue and may produce a mortality rate of approximately $90 \% .{ }^{[2]}$ IA has emerged as an important disease in recent decades due to the use of aggressive immunosuppressive therapy causing prolonged neutropenia in the treatment of cancer and leukaemia. Despite aggressive antifungal chemotherapy, death due to IA usually results 7-14 days after diagnosis. ${ }^{[3]}$

Amphotericin B, azoles, such as itraconazole, and an emerging array of inhibitors of cell-wall biosynthesis play a key role in combating fungal infection. However, such treatment is not always effective, although expensive, and there is a clear requirement for the identification of new fungal targets and antifungal therapies. ${ }^{[4]}$ The near availability of the complete $A$. fumigatus genome sequence ${ }^{[5]}$ (http://www.tigr.org) has both stimulated significant interest in, and facilitated the search for, such novel fungal targets.

Non-ribosomal peptide (NRP), polyketide (PK), fatty acid and lysine biosyntheses in many bacteria and fungi require posttranslational phosphopantetheinylation of key apo-enzymes to facilitate metabolite production. 4'-phosphopantetheinyl transferases (4'-PPTase) catalyse the transfer of $4^{\prime}$-phosphopantetheine from coenzyme $A(C \circ A)$ to a conserved serine residue within the cognate apo-enzyme (either apo-NRP or PK synthetase or $\alpha$-aminoadipate reductase (AAR)). These 4'-phosphopantetheinyl prosthetic groups anchor activated intermediates and facilitate their movement between active sites within the

multienzyme complex. ${ }^{[6]}$ In lysine biosynthesis, the 4'-PPTases serve to activate AAR, which in turn can convert $\alpha$-aminoadipate to $\alpha$-aminoadipic semialdehyde, which is an essential step in lysine biosynthesis in S. cerevisiae. ${ }^{[7]}$

Sfp-type 4'-PPTases are those primarily responsible for NRPS and PKS phosphopantetheinylation, in addition to playing a key role in AAR activation. Recent work ${ }^{[8,9]}$ suggested the existence of an Sfp-type 4'-PPTase gene in A. fumigatus, which may encode a protein of $39.6 \mathrm{kDa}$ (359 amino acids) and contain identical conserved sequence motifs to the $n p g A$ gene in $A$. nidulans. Oberegger et al. ${ }^{[10]}$ have subsequently shown that $n p g A$ gene expression is essential for the production of the siderophores ferricrocin and triacetylfusarinine, and may also be required for a number of other biosynthetic pathways, including lysine and fungal-pigment biosynthesis.

Although A. fumigatus is known to produce a range of lowmolecular-weight metabolites (e.g., siderophores and toxins), many of which are likely to be formed by either polyketide or NRP synthesis, little effort has been directed towards the investigation of NRP synthesis. Haas ${ }^{[11]}$ has indicated the presence of an NRPS gene in A. fumigatus, but revealed no further details regarding gene sequence or organisation. It is well known from studies in other prokaryotic and fungal species that NRP synthesis occurs in an ATP-dependent manner through the ac-

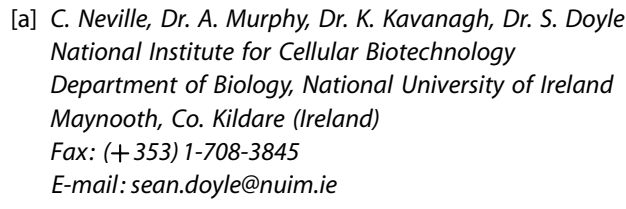


tivity of multifunctional enzymes known as non-ribosomal peptide synthetases (NRPS) and that the organisation of modules within these NRPS directly influences the order of the amino acids in the resultant peptide products. ${ }^{[12]}$ Modules within NRP synthetases are composed of domains including adenylation, thiolation (peptidyl carrier protein) and condensation domains. ${ }^{[12]}$ (Other domain types can also be present and are reviewed elsewhere. ${ }^{[13]}$ ) Each domain has a specific function: the adenylation domain recognises and adenylates, by ATP hydrolysis, a substrate amino acid, which is in turn transferred to the 4'-phosphopantetheine prosthetic group of the thiolation domain by covalent attachment to an available $\mathrm{SH}$ group on the 4'-phosphopantetheine moiety. Ultimately, the covalent attachment of the amino acid to a proximal amino acid occurs through the condensation domain followed by either sequential transfer within the NRPS modular system or release through thioesterase activity to yield functional, non-ribosomally synthesised peptides. To date, no detailed analysis of either NRPS genes or gene expression from A. fumigatus has been forthcoming, despite the emergence of the organism as a significant human pathogen.

In an effort to investigate the role of the putative 4'-PPTase gene in, and elucidate the non-ribosomal biosynthetic capacity of, A. fumigatus, we have undertaken the isolation of a $4^{\prime}$-PPTase gene, carried out evaluation of 4'-PPTase gene expression and expressed the 4'PPTase gene in a eukaryotic expression system. Secondly, we have identified and evaluated the expression of an NRPS open reading frame within the $A$. fumigatus genome. Finally, we present data that support the hypothesis that the A. fumigatus 4'-PPTase is responsible for the functional activation of an NRPS apo-enzyme through 4'-phosphopantetheinylation.

\section{Results}

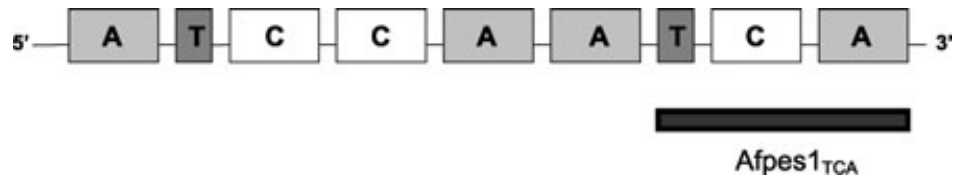

Figure 1. Schematic diagram showing the domain architecture of A. fumigatus pes 1 (Afpes1), a 14312 bp putative non-ribosomal peptide synthetase region. A: adenylation domains, $C$ : condensation domains and T: thiolation domains. The adenylation domains occur between nucleotides (amino acids (aa)) 1-912 (1-304), 4326-5505 (1442-1835), 7437-8664 (2479-2888) and 10710-11919 (3570-3973). The condensation domains occur between nucleotides (aa) 1485-2376 (495-792), 2919-3783 (973-1261) and 9336-10 161 (3112-3387). The thiolation domains encompass nucleotides (aa) 1251-1446 (417-482) and 9018-9213 (3006-3071). The 3760 bp region (termed Afpes $\left.1_{T C A}\right)$ was cloned, sequenced and expressed in the baculovirus expression system.

\section{Phylogenetic analysis of $4^{\prime}$-PPTase and NRP synthetase genes}

Data mining of the near-complete A. fumigatus genome with known sequences enabled the identification of two open reading frames (orf) potentially involved in non-ribosomal peptide synthesis.

The entire coding region $(1.12 \mathrm{~kb})$ of a $4^{\prime}$-phosphopantetheinyl transferase (4'-PPTase) was identified, cloned and sequenced (Genbank accession number AY607103). Phylogenetic analysis shows that the $A$. fumigatus 4 '-PPTase is an orthologue of the $A$. nidulans npgA/cfwA gene (Genbank accession number AF198117). The A. fumigatus 4'-PPTase was also used to BLAST (basic local alignment search tool) the National Center for Biotechnological Information (NCBI) database (http:// www.ncbi.nlm.nih.gov) in order to determine the similarity and identity of this sequence to other known 4'-PPTases. Highest similarity levels are seen to A. nidulans npgA gene which is known to be involved in penicillin biosynthesis ( $55 \%$ identity; $67 \%$ similarity) while the 4'-PPTase displays lower levels of more, the domain architecture of Afpes 1 indicates that it is a type of nonlinear (type C) NRP synthetase, as defined by at least one unusual arrangement of core A, C or T domains. ${ }^{[13]}$ The double condensation domains may allow the formation of cyclic peptides or, if present at the end of the sequence, may be involved in chain termination.

The AMP-binding domains of NRP synthetases are highly conserved and may therefore represent the specificity code of the particular NRP synthetase, as it is this domain that recognises and binds the substrate amino acid. ${ }^{[16]}$ In general, each adenylation domain contains an eight-residue, nonlinear sequence motif that is responsible for binding the substrate amino acid. The four adenylation domains of Afpes 1 were aligned with the phenylalanine-activating adenylation domain of $\mathrm{GrsA}^{[17]}$ to identify each eight-residue motif. The eight-residue motif for each domain was then used to interrogate a database of domains with assigned specificity, available at http:// raynam.chm.jhu.edu/ nrps/ by using BLAST analysis. From this alignment, the putative amino acid substrate of each adenyla- 
tion domain was determined. This analysis indicates that adenylation-domain motifs 1 (DAMVAYLS), 2 (DVKSVGAV), 3 (DAMFAGGI) and 4 (DVKSVGAV) encode $C, T, V / I$ and T/D, respectively.

\section{Expression analysis of 4'-PPTase and Afpes 1}

4'-PPTase and Afpes 1 gene expression were both assessed by Northern analysis. In addition, Afpes 1 expression was evaluated by RT-PCR. 4'-PPTase expression is evident at all time points during A. fumigatus growth from $t=24$ to $96 \mathrm{~h}$ (Figure 2). The observed band is approximately $2 \mathrm{~kb}$; this suggests that the 4 'PPTase transcript is significantly longer than the gene itself. Afpes 1 expression was only detectable at 24,48 and $72 \mathrm{~h}$ by Northern analysis (mRNA size: ca. $15 \mathrm{~kb}$ ). Afpes 1 expression at 48 (very weak) and $72 \mathrm{~h}$ was evident on RT-PCR analysis, and, despite repeated attempts, no Afpes 1 expression could be detected at $96 \mathrm{~h}$; this suggests that Afpes 1 gene expression is downregulated under the A. fumigatus culture conditions employed for these analyses. The lack of detection of Afpes 1 expression by RT-PCR at $24 \mathrm{~h}$ is suggestive of a gradual up-regulation in Afpes 1 gene expression. Alternatively, this observation could be due to the differential sensitivity of RT-PCR and Northern analysis for the detection Afpes 1 expression at low levels of transcript. rRNA loading was used as a control for the amounts of RNA used for Northern analysis and cDNA synthesis. In addition, the presence of genomic DNA was excluded by both DNAse treatment of isolated RNA prior to RT-PCR and analysis of the size difference between the genomic and CDNA amplicon of a gstA gene ${ }^{[18]}$ (Genbank accession number

$t / \mathrm{h}$

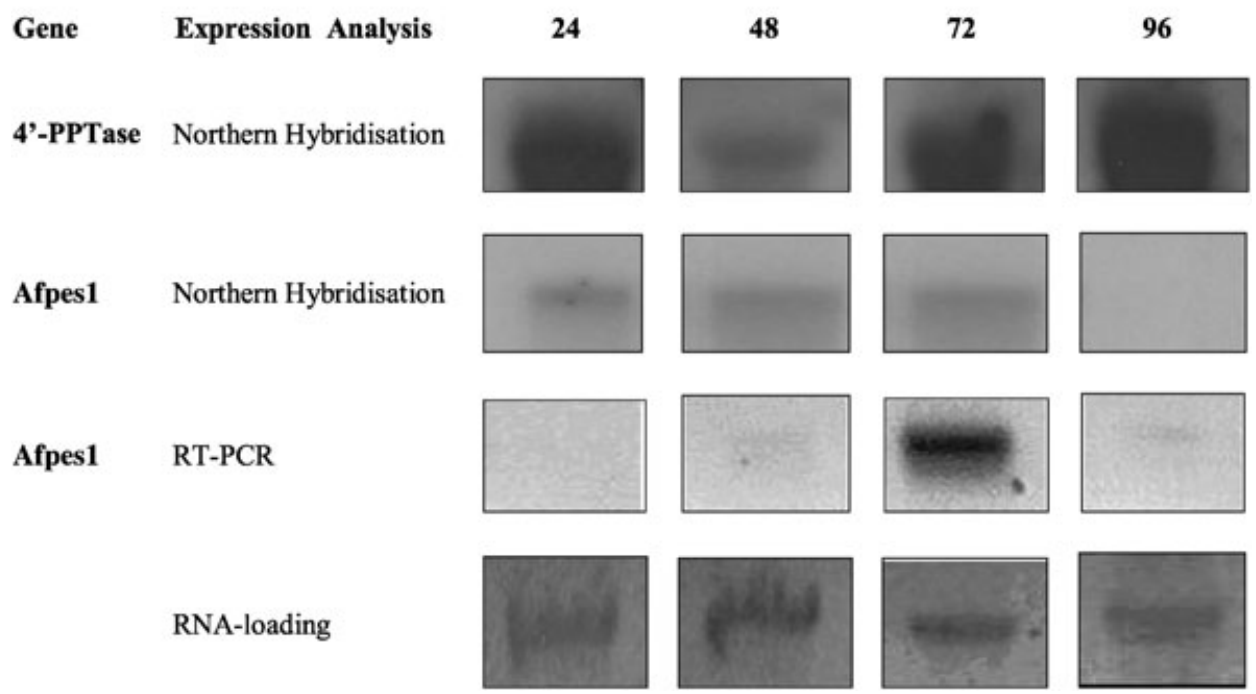

Figure 2. Gene-expression analysis of 4'-PPTase and Afpes 1 in A. fumigatus. Northern hybridisation (60 $\mu g$ total RNA per well) was used to assess 4'-PPTase and Afpes 1 expression in fungal cultures ranging from 24-96 $h$ post-inoculation. In addition, Afpes 1 expression was evaluated by RT-PCR ( $1 \mu \mathrm{g}$ total RNA per CDNA synthesis reaction) over an identical time period. Culture stationary phase was reached at $72 \mathrm{~h}$. The 4'-PPTase is constitutively expressed at all time points evaluated. AfPes 1 expression, as judged by Northern hybridisation is observed at 24, 48 and $72 \mathrm{~h}$ only. $R T-P C R$ analysis of Afpes 1 expression also detects transcripts at 48 and $72 \mathrm{~h}$. RNA from each isolation time point was applied equally for Northern hybridisation by using ribosomal RNA as loading control.
AF425746) from which introns have been excised (data not shown).

\section{Baculovirus expression of recombinant 4'-PPTase and partial NRPS (Afpes $1_{\text {TCA }}$ )}

Both 4'-PPTase- and Afpes $1_{\text {TCA }}$-encoding regions were independently transferred into a baculovirus transfer vector (pBlueBac4.5) in order to generate recombinant baculoviruses, each encoding 4'-PPTase or Afpes $1_{\text {TCA }}$, by cotransfection with linearised baculovirus. A. fumigatus 4'-PPTase was purified from recombinant baculovirus-infected insect cells by single-step $\mathrm{Ni}$ NTA agarose affinity chromatography to yield a soluble protein of $42 \mathrm{kDa}$ as judged by SDS-PAGE analysis (Figure 3). The yield of released and soluble $4^{\prime}$-PPTase was calculated to be $0.4 \mathrm{mg}$ per $10^{8}$ cells, although it was clear from subsequent analysis that significant enzyme remained within infected Spodoptera frugiperda $9\left(\mathrm{Sf}_{9}\right)$ insect cells (data not shown). MALDI-TOF mass spectrometry was used to verify the identity of the recombinant 4'-PPTase (actual/theoretical peptides detected: $8: 106$ (7.5\%), which represents $16 \%$ sequence coverage). A. fumigatus pes $1_{\text {TCA }}$ was then purified from recombinant baculovirus-infected insect cells by differential extraction and extensive washing to yield a protein of $120 \mathrm{kDa}$ as judged by SDS-PAGE analysis (Figure 3). Lower $M_{r}$ protein bands present in purified Afpes $1_{\text {TCA }}$ at approximately 38 and $62 \mathrm{kDa}$, (Figure 3, lane 3) were reactive with rabbit antisera previously raised against an E. coli-expressed condensation domain of Afpes $1_{\text {TCA }}$ and represent proteolytic fragments of the parental protein (data not shown). The yield of recombinant Afpes $1_{\text {TCA }}$ was calculated to be $2-3 \mathrm{mg}$ per $10^{8}$ cells. Although a $\mathrm{His}_{6}$ affinity tag had been engineered into the DNA construct, it was not possible to detect Afpes1 $1_{\text {TCA }}$ on Western blots with murine monoclonal anti-His $_{6}$ antibody (data not shown). Consequently, expression of recombinant Afpes $1_{\text {TCA }}$ was confirmed by both MALDITOF mass spectrometry (actual/ theoretical peptides detected: 23:287 (8\%) representing 25\% sequence coverage) and rabbit antisera previously raised against an E. coli-expressed condensation domain also encoded by Afpes $1_{\text {TCA }}$ expressed in insect cells (data not shown).

\section{Functional analysis of 4'-phos- phopantetheinyl transferase}

Functional 4'-PPTase activity was initially investigated by using an $\alpha$-AAR-activation assay previously described for the assessment of C. albicans Lys5p, which ex- 
A) $k$

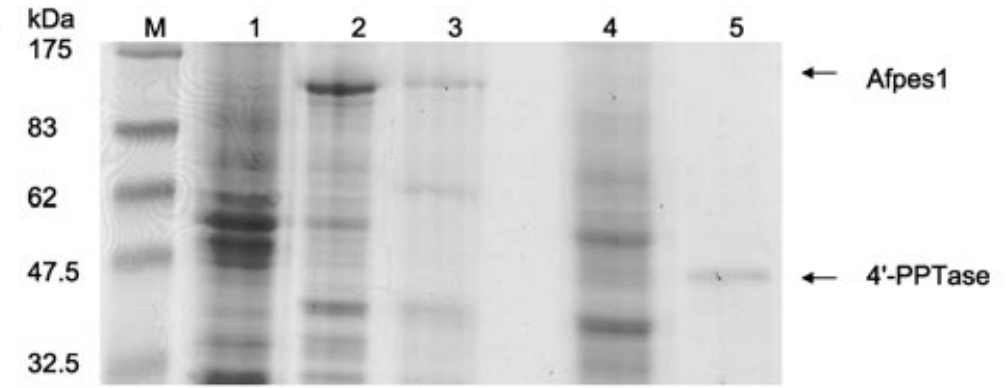

B)

$\mathrm{kDa}$
83
62
47.5
32.5
25

2

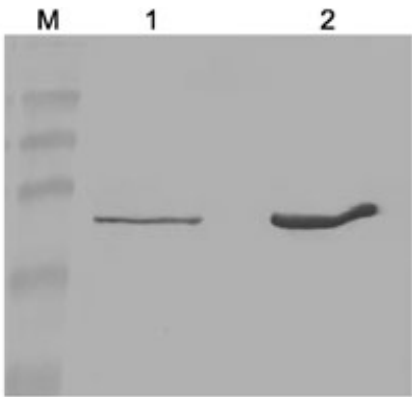

Figure 3. A) SDS-PAGE analysis of recombinant 4'-PPTase and Afpes $1_{T C A}$ from A. fumigatus. Lane M: molecular-weight markers, lane 1: uninfected insect cell lysate $\left(2.5 \times 10^{5}\right.$ cell equivalents), lane 2: Afpes $1_{T C A}$ recombinant baculovirus-infected $S f_{9}$ cells at an MOI 15, lane 3: purified Afpes $1_{T C A}$, lane 4: 4'-PPTase recombinant baculovirus-infected $\mathrm{Sf}_{9}$ cells at an $\mathrm{MOI} 15$ and lane 5: affinity-purified 4'-PPTase. In lane 3, the protein bands evident at 38 and $62 \mathrm{kDa}$ were reactive with antisera raised against the condensation domain in Afpes $1_{T C A}$ (data not shown). B) Western blot analysis of recombinant $4^{\prime}$-PPTase probed with anti-(His) ${ }_{6}$ monoclonal antibody. Lane M: molecular-weight markers, lane 1: 4'-PPTase recombinant baculovirus-infected Sf ${ }_{9}$ cells at an MOI 15 and lane 2: affinity-purified 4'-PPTase.

hibits 4'-PPTase activity. ${ }^{[7]}$ Figure 4 clearly shows that AAR activity is detectable in the presence of recombinant Lys $2 p$ and C. albicans CLD2 (Lys2p mutant) extract, which contains a func-

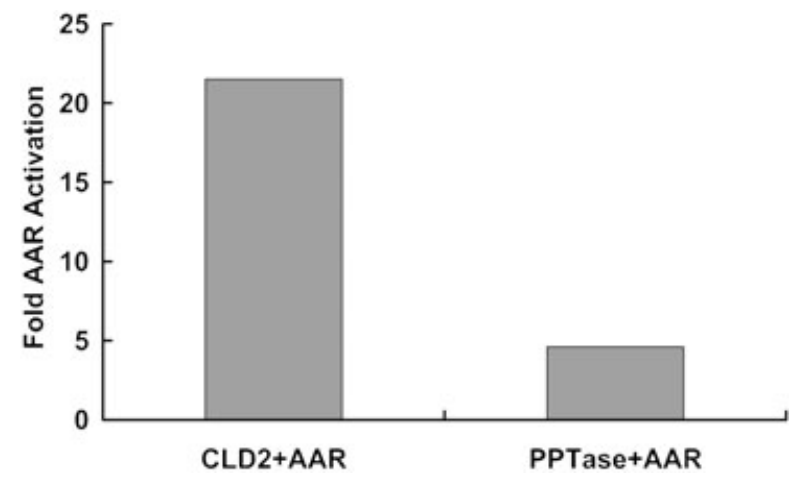

Figure 4. $\alpha$-aminoadipate reductase (AAR) activation by recombinant 4'-PPTase from A. fumigatus. Activation of C. albicans $L y s 2 p$ (apo-AAR), by a control extract (CLD2) containing a $\mathrm{C}$. albicans 4'-PPTase, resulted in a 21-fold increase in observed AAR activity. Enhancement of AAR activity (4.5-fold) is evident after prior incubation of the $\mathrm{C}$. albicans Lys $2 p$ with the A. fumigatus 4'-PPTase and represents $21.3 \%$ of positive control CLD2-induced activity. All reactions were carried out in duplicate and in the presence of $200 \mu \mathrm{m}$ acetyl CoA.

tional 4'-PPTase. When AAR activity is measured after prior incubation of the recombinant C. albicans Lys $2 p$ with the putative $A$. fumigatus 4 '-PPTase, a significant 4.5 -fold enhancement of AAR activity is evident ( $21.3 \%$ of positive control CLD2 activity). This observation confirms that the recombinant 4'-PPTase can activate C. albicans Lys $2 p$ in the presence of CoA and suggests a possible role for 4'-PPTase in lysine biosynthesis in A. fumigatus. Appropriate compensation for background absorbance (due to CLD2, 4'-PPTase and Lys2p) has been made in each case, as it was observed that protein addition contributed to background signals.

\section{NRPS 4'-phosphopantetheinylation by 4'-PPTase}

The capacity of recombinant 4'-PPTase to activate non-ribosomal peptide synthetases was investigated by coincubation of 4'-PPTase, CoA and recombinant Afpes $1_{\text {TCA, }}$ which encodes a putative thiolation domain (Afpes1 aa residues: 3006-3071), followed by MALDI-TOF mass spectrometric analysis of the reaction mixture to detect evidence of $4^{\prime}$-phosphopantetheinylation. Codigestion of the reaction mixture with both trypsin and V8 protease was used as trypsin-only digestion generated peptides from the Afpes $1_{\text {TCA }}$ thiolation domain that were too large for reliable analysis (data not shown). In addition, solutionphase enzymatic digestion was employed to optimise peptide recovery prior to MALDI-TOF mass spectrometry. It can be seen from Figure 5 that a peptide with a monoisotopic $m / z$ value of 1009.1, representing 4'-phosphopantetheinylated SFSAMK (theoretical $m / z=1009.3$ ), is detected by this analysis, thereby confirming that recombinant $A$. fumigatus $4^{\prime}$-PPTase both recognises and 4'-phosphopantetheinylates the thiolation domain present in Afpes1. In the absence of either CoA or recombinant Afpes $1_{\text {TCA }}$, no modified peptide is detected at the predicted $m / z$ ratio.

\section{Discussion}

Here we report the cloning and functional expression of the full-length open reading frame encoding an Aspergillus fumigatus 4'-phosphopantetheinyl transferase in the baculovirus-expression system. In addition, an NRP synthetase in A. fumigatus (Afpes1), which exhibits unusual domain architecture showing characteristics of a nonlinear NRP synthetase, has been identified from A. fumigatus contig 4944. A region of this NRP synthetase ( $3.7 \mathrm{~kb}$; Afpes $1_{\mathrm{TCA}}$ ) was expressed and found to undergo phosphopantetheinylation to the holo-enzyme form following activation by $4^{\prime}$-PPTase in the presence of CoA; this supports the hypothesis that the 4'-PPTase functions to activate an NRP synthetase by 4'-phosphopantetheinylation.

The A.fumigatus $4^{\prime}$-PPTase that has been investigated is identical to that previously noted by Mootz et al. and Keszenman-Pereyra et al. ${ }^{[8,9]}$ Although both previous reports speculated on the function of the 4'-PPTase gene, by comparison to the $A$. nidulans orthologue $(n p g A)$, definite confirmation that the A. fumigatus 4'-PPTase was expressed in vivo and functioned as hypothesised, in vitro, has not been available until now. From phylogenetic analysis, the A.fumigatus 4'-PPTase was confirmed to be an orthologue of the A. nidulans 4'- 


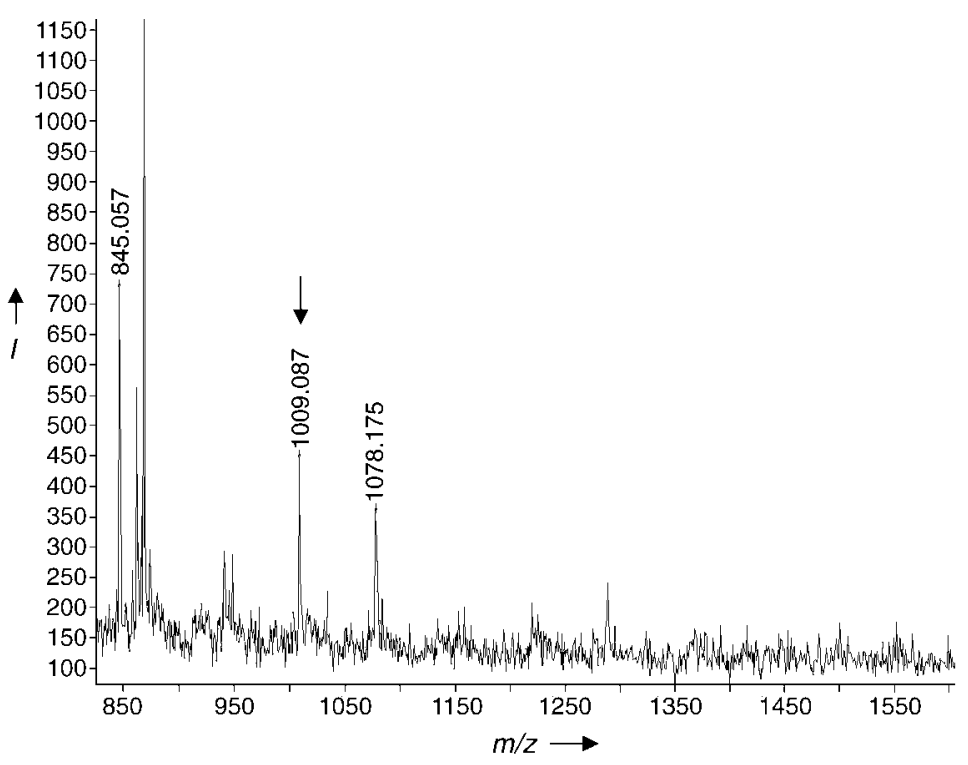

Figure 5. MALDI TOF MS analysis of in vitro phosphopantetheinylation by A. fumigatus 4'PPTase. Mass spectrum of Afpes $1_{\text {TCA }}$-derived peptide mixture (following codigestion with trypsin and V8 protease). A specific peptide with a monoisotopic $\mathrm{m} / \mathrm{z}$ value of 1009.1 is evident and represents phosphopantetheinylated-SFSAMK. In the absence of either 4'-PPTase or COA , during the enzymatic reaction, no modified peptide is subsequently detected at the expected $\mathrm{m} / \mathrm{z}$ ratio.

PPTase; this suggested common ancestry, and it also showed similarity to a Bacillus subtilis 4'-PPTase. Moreover, A. fumigatus 4'-PPTase exhibits $25 \%$ identity ( $37 \%$ similarity) to the human 4'-PPTase (Genbank accession number BC015470) and shares some significant regions of homology (WxLKExxxK) as previously noted. ${ }^{[9]}$

In most organisms there is an individual 4'-PPTase for each function, however the possible presence of only one 4'-PPTase in A. fumigatus is not unique, since recent work has identified and characterised a human 4'-PPTase that appears to exhibit a broad specificity for all 4'-phosphopantetheinylation reactions including human (apo-ACP domain for cytosolic fatty acid synthetase (FAS), mitochondrial ACP, $\alpha$-aminoadipate semialdehyde dehydrogenase activation (lysine catabolism)) and nonhuman (B. subtilis ACP-A (involved in fatty acid synthesis) and $B$. brevis tyrocidine synthetase) apo-enzymes ${ }^{[19,20]}$ A new type of 4'-PPTase was also identified in Pseudomonas aeruginosa that showed association with fatty acid synthesis and siderophore metabolism. ${ }^{[19]}$

Northern analysis of A. fumigatus 4'-PPTase expression indicates that the gene is constitutively expressed; this strongly suggests that protein $4^{\prime}$-phosphopantetheinylation is required for both primary- and secondary-metabolite production in A. fumigatus. Indeed, the putative role of 4'-PPTase in lysine biosynthesis (see below), and its role in NRP-synthetase activation is compatible with this observation.

To date, characterisation of yeast and fungal 4'-PPTases has primarily focussed on genetic complementation studies in either S. cerevisiae ${ }^{[8]}$ or A. nidulans ${ }^{[9]}$ as opposed to direct biochemical studies. Guo and Bhattacharjee have developed an AAR-activation assay to assess $4^{\prime}$-PPTase activity in vitro. ${ }^{[7]}$
Thus, the potential role of A.fumigatus 4 '-PPTase in lysine biosynthesis was investigated. The in vitro activation of $C$. albicans Lys $2 p$ (Apo-AAR) by A. fumigatus 4'-PPTase suggests that the enzyme may play a role in lysine biosynthesis. Although equivalent AAR activity (4.5-fold activation) in comparison to the positive control (22-fold activation) was not evident, this could be accounted for by differences between core 1,2 and 3 conserved motifs ${ }^{[7]}$ in C. albicans Lys5p (4'PPTase) and those present in the A.fumigatus $4^{\prime}$ PPTase. Moreover, phylogenetic analysis confirms that the 4'-PPTase encoded by C. albicans clusters to a separate clade to that of the A. fumigatus 4'-PPTase.

A number of groups have reported the application of protein mass spectrometry for the determination of apo-enzyme $4^{\prime}$-phosphopantetheinylation. ${ }^{[20,22]}$ In these reports, the substrate proteins exhibited molecular masses in the order of $11-38 \mathrm{kDa}$; however, in the case of baculovirus-expressed Afpes $1_{\text {TCA }}$ $(120 \mathrm{kDa})$, there was concern that protein mass spectrometry would not exhibit the required resolution to confirm 4'-phosphopantetheinylation. Consequently, the post-phosphopantetheinylation reaction mixture was enzymatically digested (with trypsin and V8 protease) prior to peptide mass fingerprinting. As the expected peptide containing the conserved serine $(\underline{S})$ within the thiolation domain (SFSAMK) had $m / z=670.3 \mathrm{Da}$, post-translational modification by the 4'-PPTase was expected to add an additional $339 \mathrm{Da}$ and result in a peptide of $\mathrm{m} / \mathrm{z}=$ 1009.3 Da. Our data confirm the presence of the 1009.3 Da peptide following coincubation of CoA, 4'-PPTase and Afpes $1_{T}$ CA under optimal conditions. The confirmation that 4'-PPTase can 4'-phosphopantetheinylate a thiolation domain of an NRP synthetase is highly significant as it strongly indicates that at least one NRP synthetase encoded by the A.fumigatus genome can be activated, and thereby participate in non-ribosomal peptide synthesis in the organism. Until now, no mechanistic evidence of NRP synthesis in A. fumigatus has been forthcoming, despite the existence of multiple siderophores and other low-molecular-weight metabolites. Although it is unlikely that Afpes 1 encodes a siderophore (see below), we hypothesise that $4^{\prime}$-PPTase expression and functionality will also be essential for siderophore production in A. fumigatus and so may represent a key drug target in controlling siderophore production with a view to minimising organism virulence. ${ }^{[10,11]}$ Moreover, the identification of only one 4'-PPTase in A. fumigatus, to date, that may be involved in multiple 4'-phosphopantetheinylation reactions, makes selective inhibition in the absence of concomitant inhibition of human 4'-PPTase even more attractive with a view to anti- $A$. fumigatus drug therapy in humans. However, the identification of similar enzymes in A. fumigatus cannot be completely ruled out at present.

The Afpes 1 open reading frame, which extends over $14300 \mathrm{bp}$, encodes the first NRP synthetase (predicted protein relative molecular mass: $460 \mathrm{kDa}$ ) to be definitively identified in A. fumigatus. Furthermore, Northern analysis confirms that the Afpes1 gene is differentially expressed (transcript size: ca. 
$15 \mathrm{~kb})$ at 24,48 and $72 \mathrm{~h}$ post-inoculation when A. fumigatus is grown in liquid culture $(5 \%, v / v$ FCS in minimal essential medium (MEM)). Upon phylogenetic analysis, Afpes1 clusters with the NRP synthetase (AbrePsy1) from Alternaria brassi$\mathrm{cae}^{[15]}$ thus suggesting orthology. Also grouped in the same clade is the pesA gene of Metarhizium anisopliae. ${ }^{[14]}$ Unfortunately the functions of the NRP synthetase genes of both A. brassicae and M. anisopliae have yet to be identified, but are proposed to be involved in destruxin biosynthesis, which is thought to play a role in the progression of plant disease mediated by both species. ${ }^{[14,15]}$ Although it has been proposed that the NRP synthetase gene of $A$. brassicae may play a role in siderophore biosynthesis, ${ }^{[15]}$ particularly since the first adenylation domain of AbrePsy1 is predicted to encode 5-hydroxyornithine, the clustering of Afpes 1 away from a putative Aspergillus NRP synthetase (A. oryzae sid2; Genbank accession number AB087617) suggests that this may not, in fact, be the case. A further difference between both A.fumigatus and Alternaria genes is that while the Afpes 1 gene represents a complete exonic region, the AbrePsy1 gene contains seven introns. Thus, while it is possible that Afpes 1 may have a similar function to that of AbrePsy1, orthology does not guarantee similarity of function, which can change over time. ${ }^{[23]}$

One striking observation with respect to the structure of Afpes 1 is the arrangement of the individual domains within the protein: ATCCAATCA. Such NRPSs are referred to as nonlinear (type C), as recently described. ${ }^{[13]}$ The presence of an adenylation domain at the $C$ terminus of an NRP synthetase is somewhat unusual and suggests that Afpes 1 may be involved in a multisubunit NRPS complex with another synthetase. Interestingly, the production of ergot alkaloids containing D-lysergic acid in the fungus Claviceps purpurea occurs through a cluster of synthetases, namely LPS1 $(370 \mathrm{kDa})$ and LPS2 $(140 \mathrm{kDa})$. These synthetases are encoded by two genes, cpps2 and cpps 1, present in a gene cluster. Mechanistically, it has been shown that D-lysergic acid is initially bound to LPS2 through a thioester bond and that this thioester-bound D-lysergic acid is then transferred to LPS1. Following this, three condensation reactions into the D-lysergyl mono-, di- and, finally, tripeptide thioester occur to result in the formation of $D$ lysergyl tripeptide lactam. This system of ergot-peptide formation was the first identified fungal-NRP-synthesis system consisting of different NRP synthesis subunits. ${ }^{[2]}$ Other examples of type C NRP synthetases include PesA of Metarhizium anisolpiae and AbrePsy1 of Alternaria brassicae. ${ }^{[14,15]}$

The functional assessment of 4'-PPTase activity and the identification of a novel NRP synthetase provide new insights into non-ribosomal peptide synthesis in A.fumigatus. In addition, we postulate that the $4^{\prime}$-PPTase may also play a role in lysine production within A. fumigatus by activation of key biosynthetic enzymes. Although no definitive role for the NRP synthetase, Afpes1, has been identified, it may be responsible for toxin biosynthesis, and work is ongoing in our laboratory to identify gene function within the ascomycete. Modulation of 4'-PPTase expression or functionality in A. fumigatus may represent a novel antifungal target.

\section{Experimental Section}

Microorganisms and culture media: Aspergillus fumigatus strain ATCC 26933 (obtained from the American Type Culture Collection, Manasas, VA (USA)) was used for this work and was grown at $37^{\circ} \mathrm{C}$ in MEM supplemented with foetal calf serum (FCS; $5 \%, v / v)$ for $48 \mathrm{~h}$ in order to facilitate DNA isolation. For gene-expression studies, in which culture was extended to $96 \mathrm{~h}$, it was observed that stationary phase was reached after $72 \mathrm{~h}$ post-inoculation. Escherichia coli strains Top10', XL1Blue (Invitrogen, Dublin (Ireland)) and BL21 (Novagen, Nottingham (UK)) were grown on LB agar (SigmaAldrich, Dorset (UK)) and LB agar containing ampicillin (100 $\mu \mathrm{g} \mathrm{mL}^{-1}$-chloramphenicol $\left(34 \mu \mathrm{g} \mathrm{mL}^{-1}\right)$ was also included for BL21 culture), where appropriate, at $37^{\circ} \mathrm{C}$ overnight.

Bioinformatic analyses, genomic DNA isolation and PCR cloning: Preliminary A. fumigatus sequence data were obtained from The Institute for Genomic Research website at http://www.tigr.org. By using the sequence search facility at http://www.tigr.org, the nearcomplete $A$. fumigatus genome was interrogated by using enniatin synthetase (Genbank accession number Z18755), with a BLAST program. Genomic DNA was isolated by crushing A. fumigatus in liquid $\mathrm{N}_{2}$ followed by phenol/chloroform extraction, washing with ethanol $(70 \%, v / v)$ and final DNA resuspension in Tris- $\mathrm{HCl}(10 \mathrm{~mm})$, EDTA $(1 \mathrm{~mm}$; pH 7.4, $300 \mu \mathrm{L})$. Genomic DNA $(5 \mu \mathrm{L})$ was used in all PCR reactions containing Tris $\mathrm{HCl}(10 \mathrm{~mm} ; \mathrm{pH} 9.0), \mathrm{KCl}(50 \mathrm{~mm})$, Triton X-100 $(0.1 \%, v / v), \mathrm{MgCl}_{2}(1.5 \mathrm{~mm})$, dNTP $(200 \mu \mathrm{M}$ of each), forward and reverse primer (1.0 $\mu \mathrm{M}$ of each; Afpes1-F: $5^{\prime}$-GAGAGGTACCATGTCGGAAGCAACACG-3' and Afpes1-R: 5'-GAGAGGTACCTTACCAGTCAGCCTC-3'; or 4'-PPTase-F: 5'-GAGAGGATCCATGGGCTCTGCACAAAACG-3' and 4'-PPTase-R: 5'-GAGAAAGCTTGGGCTGTTाTTTTATACAC- $3^{\prime}$ ) and Taq polymerase (1 unit) (Promega, Southampton (UK)) in a total volume of $49.5 \mu \mathrm{L}$. AccuTaq LA DNA polymerase (Sigma-Aldrich) was used to amplify a partial region from Afpes 1 only. After incubation at $95^{\circ} \mathrm{C}$ for $5 \mathrm{~min}$, all 4'-PPTase and Afpes 1 PCR reactions were continued with 35 cycles consisting of $60 \mathrm{~s}$ denaturation ( $90 \mathrm{~s}$ for Afpes 1 ) at $95^{\circ} \mathrm{C}$, primer annealing at $55^{\circ} \mathrm{C}$ for $60 \mathrm{~s}$, extension at $72^{\circ} \mathrm{C}$ for $240 \mathrm{~s}$ and finally extension for $360 \mathrm{~s}$ at $72{ }^{\circ} \mathrm{C}$. PCR-amplified DNA was analysed by agarose gel electrophoresis whereby product $(10 \mu \mathrm{L})$ was electrophoresed on agarose $(1 \%, w / v)$ containing ethidium bromide $\left(0.5 \mu \mathrm{g} \mathrm{mL}^{-1}\right)$ for $30 \mathrm{~min}$ at $100 \mathrm{~V}$. Visualisation of the 4'-PPTase- and Afpes1-derived amplicons was performed by using an "Eagle-Eye II" digital still video system. Once PCR had been successfully carried out, both amplicons were individually cloned into the TOPO cloning vector and subsequently transferred into separate pBlueBac4.5 vectors (Clontech, Palo Alto, CA) by using standard molecular biology techniques, for expression in $\mathrm{Sf}_{9}$ cells.

Northern hybridisation and RT-PCR analysis of $4^{\prime}$-PPTase and Afpes 1 gene expression A. fumigatus cultures were harvested at defined time points $(24,48,72$ and $96 \mathrm{~h})$, mycelia collected, washed with ice-cold phosphate-buffered saline and total-RNA extracted by using TRI reagent (Sigma-Aldrich). Northern analyses ( $60 \mu \mathrm{g}$ total RNA per well) were carried out by using ${ }^{32} \mathrm{P}$-labelled regions of Afpes1 (NT: 9716-10535) and 4'-PPTase (NT: 1-1123) to detect relevant transcripts according to standard protocols. ${ }^{[25]}$ RTPCR was performed by following CDNA synthesis with Superscript (Promega; $1 \mu \mathrm{g}$ RNA per reaction) and relevant PCR primers (see above).

Construction of recombinant baculovirus encoding 4'-PPTase and Afpes $1_{\text {TCA }}: \mathrm{Sf}_{9}$ insect cells were co-transfected with recombinant transfer vectors and Bsu36 I-linearised BacPAK-6 baculovirus by using Bacfectin liposomal preparation (Clontech, Palo Alto, 
CA). ${ }^{[24]}$ Well plates containing $\mathrm{Sf}_{9}$ cells were infected with the cotransfection mixtures, and the TC-100 supernatant was harvested after 5 days' incubation at $27^{\circ} \mathrm{C}$. Pure clones of either $4^{\prime}$-PPTase- or Afpes $1_{\text {TCA }}$-encoding recombinant baculoviruses were identified by plaque assay. ${ }^{[26]}$

Antigen expression and purification: Recombinant baculoviruses encoding the 4'-PPTase and Afpes $1_{\text {TCA }}$ genes were individually used to infect $\mathrm{Sf}_{9}$ cells in monolayer culture at a multiplicity of infection of 15 . Infected cells $\left(5 \times 10^{8}\right)$ were harvested 4 days post-infection. In each case, SDS-PAGE, Western Blotting and MALDI-TOF mass spectrometry were used to confirm the presence of 4'-PPTase and Afpes $1_{\text {TCA }}$. It was observed that while the 4 '-PPTase was soluble and could be isolated from cell pellets by washing with PBS, followed by $\mathrm{Ni}$ chelate affinity chromatography by elution with imidazole $(200 \mathrm{~mm})$, a more extensive extraction procedure was required for Afpes $1_{\text {TCA }}$ purification. For Afpes $1_{\text {TCA }}$ isolation, cells were lysed in the presence of protease inhibitors (phenylmethylsulfonyl fluoride $(0.1 \mathrm{~mm})$, pepstatin $\left(2 \mu \mathrm{g} \mathrm{mL}^{-1}\right)$ and leupeptin $\left.\left(2 \mu \mathrm{g} \mathrm{mL}^{-1}\right)\right)$ by the addition of phosphate-buffered saline/sodium deoxycholate $(0.5 \%, w / v)$ and subjected to DNAse (Sigma, Poole (UK)) treatment (final concentration: $10 \mu \mathrm{g} \mathrm{mL}^{-1}$ ), and the insoluble pellet was washed extensively to remove contaminating proteins. ${ }^{[26]}$ Insoluble Afpes $1_{\text {TCA }}$ was resuspended in guanidinium thiocyanate $(0.5-$ $1.0 \mathrm{~mL}, 6 \mathrm{M})$, containing dithiothreitol $(5 \mathrm{~mm})$, at a concentration of $3 \mathrm{mg} \mathrm{mL}^{-1}$ and sequentially dialysed against sodium carbonate $(50 \mathrm{~mm}$; pH 9.4) containing Urea ( $3 \mathrm{M})$ and sodium carbonate (50 mм; pH 9.4) to produce soluble protein.

Enzyme assays: AAR activation by recombinant 4'-PPTase was determined as described by Guo et al. and Guo and Bhattacharjee ${ }^{[5,25]}$ Briefly, restoration of AAR activity by $4^{\prime}$-phosphopantetheinylation in apo-Lys $2 p$ from C. albicans is indicative of 4'-PPTase activity. Therefore, recombinant $A$. fumigatus $4^{\prime}$-PPTase activity was assessed by incubating the enzyme $(50 \mu \mathrm{g}$ per reaction) with recombinant Lys $2 p(100 \mu \mathrm{g}$; final volume $500 \mu \mathrm{L})$ followed by detection of AAR activity by determination of $\triangle A 460 \mathrm{~nm}$ due to the conversion of $\mathrm{DL}-\alpha$-aminoadipate to $\alpha$-aminoadipate- $\delta$-semialdehyde. C. albicans CLD2 lysate (a Lys2p mutant expressing C. albicans 4'PPTase) containing $1 \mathrm{mg}$ total protein was used as a positive control for the reaction. NRPS 4'-phosphopantetheinylation was determined by incubating Afpes $1_{\mathrm{TCA}}(100 \mu \mathrm{g})$ and 4 '-PPTase $(10 \mu \mathrm{g})$ in the presence of $\mathrm{CoA}(100 \mu \mathrm{M} \text {; reaction volume } 100 \mu \mathrm{L})^{[26,27]}$ followed by urea denaturation ( $5 \mathrm{M}$ final) and in-solution double digestion with trypsin and S. aureus V8 protease prior to MALDI-TOF peptide mass fingerprinting to detect phosphopantetheinylated peptide species.

MALDI-TOF mass spectrometry: Mass spectrometry was carried out by using an Ettan MALDI-TOF mass spectrometer (Amersham Biosciences (Europe), Freiburg (Germany)). Protein samples for peptide mass determination were either i) separated by SDS-PAGE, digested with trypsin or ii) obtained following in-solution enzymatic digestion and deposited $(1 \mu \mathrm{L})$ with $\alpha$-cyano-4-hydroxycinnaminic acid ( $1 \mu \mathrm{L}$; acetonitrile in aqueous trifluoroacetic acid $5 \mathrm{mg} / 200 \mu \mathrm{L}$ $50 \%, v / v)$ onto mass spectrometry slides and allowed to dry prior to delayed extraction and reflectron TOF analysis at $20 \mathrm{kV}$.

\section{Acknowledgements}

This work was funded under the Irish Higher Education Authority-Programme for Research in Third Level Institutions (HEAPRTLI) Cycle 3. C.N. was a recipient of a Daniel O'Connell Fellowship from NUI Maynooth. Preliminary sequence data were ob- tained from The Institute for Genomic Research website at http:// www.tigr.org. Sequencing of Aspergillus fumigatus was funded by the National Institute of Allergy and Infectious Disease U01 Al48830 to David Denning and William Nierman, the Wellcome Trust, and Fondo de Investicagiones Sanitarias. Finally we are grateful to Prof. J. K. Bhattacharjee, Miami University, Ohio (USA) for AAR reagents.

Keywords: drug targets $\cdot$ mycotoxin $\cdot$ non-ribosomal peptide synthetases $\cdot$ polyketides $\cdot$ siderophores

[1] J. L. Brookman, D. W. Denning, Curr. Opin. Microbiol. 2000, 3(5), 468 474.

[2] P. Daly, K. Kavanagh, Br. J. Biomed. Sci. 2001, 58, 197- 205.

[3] D. W. Denning, Clin. Infect. Dis. 1996, 23, 608-615.

[4] D. P. Kontoyiannis, G. P. Bodey, Eur. J. Clin. Microbiol. Infect. Dis. 2002, 21 (3), $161-172$

[5] J. E. Mabey, M. J. Anderson, P. F. Giles, C. J. Miller, T. K. Attwood, N. W. Paton, E. Bornberg-Bauer, G. D. Robson, S. G. Oliver, D. W. Denning, Nucleic Acids Res. 2004, 32, $401-405$

[6] R. H. Lambalot, A. M. Gehring, R. S. Flugel, P. Zuber, M. LaCelle, M. A Marahiel, R. Reid, C. Khosla, C. T. Walsh, Chem. Biol. 1996, 3, 923-936.

[7] S. Guo, J. K. Bhattacharjee, FEMS Microbiol. Lett. 2003, 224, 261-267.

[8] H. D. Mootz, K. Schörgendorfer, M. A. Marahiel, FEMS Microbiol. Lett. 2002, 213, $51-57$.

[9] D. Keszenman-Pereyra, S. Lawrence, M. E. Twfieg, J. Price, G. Turner, Curr. Genet. 2003, 43, 186-190.

[10] H. Oberegger, M. Eisendle, M. Schrettl, S. Graessle, H. Haas, Curr. Genet. 2003, 44, $211-215$

[11] H. Haas, Appl. Microbiol. Biotechnol. 2003, 62, 316-330.

[12] H. Kleinkauf, H. von Dohren, Eur. J. Biochem. 1996, 236, 335-351.

[13] H. D. Mootz, D. Schwarzer, M. A. Marahiel, ChemBioChem 2002, 3, 490 504.

[14] A. M. Bailey, M. J. Kershaw, B. A. Hunt, I. C. Paterson, A. K. Charnley, S. E. Reynolds, J. M. Clarkson, Gene 1996, 173, 195-197.

[15] T. Guillemette, A. Sellam, P. Simoneau, Curr. Genet. 2004, 45, 214-224.

[16] H. von Döhren, U. Keller, J. Vater, R. Zocher, Chem. Rev. 1997, 97(7), $2675-2706$

[17] T. Stachelhaus, H. D. Mootz, M. A. Marahiel, Chem. Biol. 1999, 6, $493-$ 505.

[18] J. A. Fraser, M. A. Davis, M. J. Hynes, Appl. Environ. Microbiol. 2002, 68, 2802-2808.

[19] V. Praphanphoj, K. A. Sacksteder, S. J. Gould, G. H. Thomas, M. T. Geraghty, Mol. Genet. Metab. 2001, 72, 336-342.

[20] A. K. Joshi, L. Zhang, V. S. Rangan, S. Smith, J. Biol. Chem. 2003, 278, $33142-33149$.

[21] R. Finking, J. Solsbacher, D. Konz, M. Schobert, A. Schafer, D. Jahn, M. A. Marahiel, J. Biol. Chem. 2002, 277, $50293-50302$.

[22] K. J. Weissman, H. Hong, M. Oliynyk, A. P. Siskos, P. F. Leadlay, ChemBioChem 2004, 5, 116-125.

[23] D. B. Searls, Nature Rev. 2003, 2, 613-623.

[24] T. Correia, N. Grammel, I. Ortel, U. Keller, P. Tudzynski, Chem. Biol. 2003, $10,1281-1292$.

[25] J. Sambrook, E. F. Fritsch, T. Maniatis, Molecular Cloning-A Laboratory Manual, 2nd ed., Cold Spring Harbor Laboratory Press, New York, 1989.

[26] O. Ennis, A. Corcoran, K. Kavanagh, B. P. Mahon, S. Doyle, J. Clin. Virol. 2001, 22, 55-60.

[27] S. Guo, S. A. Evans, M. B. Wilkes, J. K. Bhattacharjee, J. Bacteriol. 2001, 183, 7120-7125.

[28] L. Du, B. Shen, Chem. Biol. 1999, 6, 507-517.

[29] C. Sanchez, L. Du, D. J. Edwards, M. D. Toney, B. Shen, Chem. Biol. 2001, $8,725-738$

Received: May 10, 2004

Revised: December 2, 2004

Published online on February 18, 2005 